\title{
State Estimation of Congested TCP Traffic Networks
}

\author{
Atulya Nagar, Ghulam Abbas, and Hissam Tawfik \\ Intelligent and Distributed Systems Laboratory, Deanery of Business and Computer Sciences, \\ Liverpool Hope University, Liverpool, L16 9JD. UK. \\ \{nagara, tawfikh\}@hope.ac.uk
}

\begin{abstract}
State Estimation is an intrinsic element of many network management systems, like Power Distribution Networks and Water Distribution Networks, where its implementation not only facilitates real-time online monitoring with better observability, but it also enables an advanced control with improved system security. This paper presents a new technique based on State Estimation to address some general shortcomings of the current Active Queue Management schemes such as RED and discusses potential issues in TCP networks in order to achieve better performance.
\end{abstract}

\section{Introduction}

Congestion typically refers to a situation when a TCP service either fails to fulfill a request to transfer a bulk of data, or it ends up with extensive service delays. Furthermore, data packets may also be lost in an attempt to complete the request. If the congestions are not dealt with appropriately, the packet loss rate becomes high enough, giving rise to retransmissions of lost packets and consequently cause further service delays. The Transmission control protocol (TCP) has been designed exclusively to offer a reliable service in terms of data delivery. Early implementations of TCP led to, what was known as "congestion-collapse", in which a network failed to respond altogether. This situation was soon overcome by more reliable TCP implementations [2]. However, the rapid increase in users around the globe, with a consequent increase in data requirements, has offered many threats to this reliability. These limitations are studied and remedied in this paper, by applying State Estimation mainly due to the following reasons. At first, no mathematical model is perfect and therefore may not capture all behavioral aspects of the actual physical state of the system. Numerous effects of the underlying system are deliberately left un-modeled, while the assumptions of the modeled effects are not correct under all circumstances. As such, there may be many uncertainties present in any mathematical model. Moreover, the underlying systems are driven not only by the control inputs but are often driven by disturbances or noises which cannot be modeled deterministically. The State Estimation technique proposed in this paper uses the Kalman filtering approach to try to address the general problems of RED models. We have used the discrete time model proposed in [1], as a case study in this paper, to compare the results from our State Estimator to the Simulation results of this model. 


\section{TCP Traffic State Estimator}

The general TCP traffic flow State Estimation problem can be posed in a similar way as formulated in the Power and Water systems [6,4]. To have a more concrete description of the problem, consider the following. Let $x_{k}$ be a given signal at time step $k$ and $\mathcal{E}$ be the noise. Considering that only the sum of signal and the noise can be observed, it can be generally represented as,

$$
\boldsymbol{Z}=H X+\varepsilon
$$

Where, $\boldsymbol{Z}$ is the measurement vector which is updated at each scan. $\mathrm{X}$ is the State vector, $H$ is as Identity matrix ( $m \times n, m \geq n$ ) relates state to measurement $Z$, and $\varepsilon$ is the Vector of measurement errors. The error $\varepsilon$ can arise due to a number of situations, e.g., inaccuracy of network model, measurement noise and inaccuracy of RED. The mathematical model may serve its purpose well in most cases but the assumptions of the mathematical model are not correct in all circumstances, for example, presence of a very large number of network nodes and packet-flows may affect the calculations up to a fractional level which can accumulate into a high level of measurement uncertainty when the results are used as feedback control. Moreover, an RED that operates on a router at some congested link and uses the exponentiallyweighted-average-queue-length to predict packet losses and impose flow control may use wrong parameters (weights) and can consequently lead to uncertainties. A Kalman filter State Estimator is used here for this purpose. The expressions for the time and measurement updates of the Kalman filter, in order to devise a State Estimation algorithm, can be derived based on the treatment given in $[1,5]$.

\section{Results and Discussions}

The derived Kalman algorithm for the queue length $\boldsymbol{q}$, average queue length $\boldsymbol{X}$ and congestion window $\boldsymbol{W}$ is implemented in MATLAB. The following section presents comparison of the results from the Simulator [1] and the Estimator using the dumbbell and Y-shape topologies [3], the configurations of the congested connection listed in table 1 .

According to the law of flow conservation [3] the flow into a congested link depends on the number of packets being injected by a sender into a link and as such, the accuracy of congestion window size is of significant importance. The congestion avoidance model [1] used in this paper, increments congestion window by $1 / W$ after the receipt of each acknowledgment. While this could work well for a small number of senders, it can lead to uncertainties in the presence of a large number of senders simultaneous transmitting through a link. As the acknowledgement is modeled to arrive in one round-trip time $\left(T_{p}+q / B\right)$, which depends on the queue length $\boldsymbol{q}$ (queuing delay, $\boldsymbol{q} / \boldsymbol{B}$ ), the estimation assumes the round trip time to be corrupted by a small fraction $(0.0005 \mathrm{~s})$. This fractional change is certain to occur when there is some background traffic present i.e. the congested router is also serving some other flows arriving from other nodes (note that the model assumed no background traffic). This 
fractional inaccuracy in the round-trip time can accumulate into large inaccuracy and consequently, the congestion window measurement becomes noisy after a few round trip times. For example, assuming 30 senders simultaneously transmitting through a queue and then calculating the round-trip time, the noise robustness becomes 0.02 packets in congestion window of each sender. The noise robustness increases at the start of the congestion soon after the first packet drop between $2^{\text {nd }}$ and $3^{\text {rd }}$ second. This is because the sender waits for a relatively longer round trip time during the recovery phase (note the horizontal increase of the noisy measurements). Noise robustness also increases with the increased number of senders.

Table 1. Network parameters

\begin{tabular}{|l|l|l|}
\hline Variable & Description & Value \\
\hline $\boldsymbol{q}_{\min }$ & RED parameter & 150 \\
\hline $\boldsymbol{q}_{\max }$ & RED parameter & 300 \\
\hline $\mathrm{T}_{\mathrm{p}}$ & Propagation delay & $0.1 \mathrm{~s}$ \\
\hline $\mathrm{B}$ & Bandwidth of bottleneck link & $1 \mathrm{Mbps}$ \\
\hline$p_{\max }$ & RED parameter & 0.1 \\
\hline Weight & RED parameter & 0.001 \\
\hline
\end{tabular}

The results of estimated queue length were also compared to the results of simulated queue length [1] in the presence of certain noise. As the measurement of the queue length depends on the congestion window size of each sender transmitting through this queue $q=1 / W$, the estimator assumes five senders transmitting simultaneously, and the value of each $\boldsymbol{W}$ to be corrupted by 0.02 packets. The flow $q=\sum 1 / W$ can produce the following effects on the queue size.

Table 2 lists mean queue-length of each sender observed for 10 seconds. In the presence of noise the measurement of the simulated queue length reflects wrong values. It means that, some of the buffer space at the router's queue remains unutilized due to the noisy measurements, and the packets are dropped by the router (when queue reaches its capacity) whereas, in reality, there still remain some unoccupied space. The results from the estimator match closely with the results of

Table 2. Noise Robustness

\begin{tabular}{|c|c|c|c|}
\hline \multicolumn{4}{|c|}{ Noise Robustness } \\
\hline $\begin{array}{l}\text { No. of } \\
\text { Senders }\end{array}$ & $\begin{array}{l}\text { Simulated mean } \\
\text { Queue-length } \\
\text { (without noise) }\end{array}$ & $\begin{array}{l}\text { Simulated mean } \\
\text { Queue-length } \\
\text { (with noise) }\end{array}$ & $\begin{array}{l}\text { Estimated mean } \\
\text { Queue-Length } \\
\text { (with noise) }\end{array}$ \\
\hline 1 & 69.5827 & 70.5673 & 69.5714 \\
\hline 2 & 116.6457 & 119.3910 & 116.6333 \\
\hline 3 & 131.3254 & 137.9962 & 131.3130 \\
\hline 4 & 137.8840 & 150.1776 & 137.8696 \\
\hline 5 & 141.0444 & 160.0377 & 141.0266 \\
\hline
\end{tabular}


simulator which assumes no noise, i.e. the estimation is capable of removing the measurement noise and reflecting correct mean queue-length.

Moreover, it is also clear from the results that the measurement inaccuracy increases with the increased number of senders, consequently more packet drops will occur which in turn will lead to increased level of congestion. The accuracy of the measurements of average queue length $\boldsymbol{X}$ are of significant importance in terms of systems control, as a router using RED will drop packets as soon as $\boldsymbol{X}$ reaches $\boldsymbol{q}_{\text {min }}$. As the measurement of the average queue depends on the actual queue length $\boldsymbol{q}$, the noisy measurements of $\boldsymbol{q}$ can in turn affect the measurement of $\boldsymbol{X}$ resulting in early packet-drops prior to buffer filling.

\section{Concluding Remarks}

A State Estimation coupled with RED algorithm can provide a better control and management of the system, and security benefits.

\section{References}

1. Frommer, I., Harder, E., Hunt, B., Lance, R., Ott, E. and Yorke, J.: Two Models for the Study of Congested Internet Connections. Int. Conference on Communications and Computer Networks, (CCN 04). (2004).

2. Jacobson, V.: Modified TCP Congestion Avoidance Algorithm, Technical Report, Network Research Group LBL (1990).

3. Mathis, M. and Mahdavi, J.: Forward Acknowledgements: Refining TCP Congestion Control., in proceedings of the Int. Conf. on Applications, architectures, and protocols for computer communications, California, United States, 28-30 August (1996), Vol. 26, no. 4, p. 281-291

4. Nagar, A.K., Powell, R.S.: LFT/SDP Based Approach to the Uncertainty Analysis for State Estimation of Water Distribution Systems, IEE Journal of Control Theory and Applications, (2002) pp. 137-142, Vol. 149, issue 2.

5. Welch, G. and Bishiop, G.: An Introduction to the Kalman Filter, Technichal Report, SIGGRAPH 2001, Los Angeles Conventional Contre, (2001) 12-21.

6. Wu, F. F.: Power System State Estimation: A Survey, International Journal of EPES, Electric Power \& Energy Systems, (1990), p. 80-87. 